\title{
INVERSE PROBLEMS FOR A CLASS OF MEASURE DEPENDENT DYNAMICAL SYSTEMS
}

\author{
H. T. BANKS \\ CENTER FOR RESEARCH IN SCIENTIFIC COMPUTATION \\ BOX 8205 \\ N. C. STATE UNIVERSITY \\ RALEIGH, NC 27695-8205 \\ D. M. BORTZ \\ DEPARTMENT OF MATHEMATICS \\ UNIVERSITY OF MICHIGAN \\ 2074 EAST HALL \\ 525 EAST UNIVERSITY AVENUE \\ ANN ARBOR, MI 48109-1109
}

\begin{abstract}
We consider a class of probability measure dependent dynamical systems which arise in the study of multiscale phenomena in diverse fields such as immunological population dynamics, viscoelasticity of polymers and rubber, and polarization in dielectric materials. We develop an inverse problem framework for studying systems with distributed temporal delays. In particular, we establish conditions for existence and uniqueness of the forward problem and well-posedness (including method stability under numerical approximations) for the inverse problem of estimating the probability measures. We show that a motivating class of models of HIV infection dynamics satisfies all the conditions of our framework, thereby providing a theoretical foundation for inverse problem computations with these models.
\end{abstract}

\section{INTRODUCTION}

In this paper, we present a general theoretical framework including implementable approximation ideas for inverse problems involving measure dependent dynamical systems. In particular, we

Date: September 23, 2004.

Key words and phrases. Probability Measure Dependent Dynamics; Inverse Problems; Well-posedness; Prohorov Metric; Nonlinear Systems with Delays; Multiscale Modeling. 
treat inverse problems for systems wherein the dynamics are given for the expected values of some of the states

$$
\bar{x}(t, P)=\mathscr{E}_{P}[x(t, q)]=\int_{Q} x(t, q) d P(q),
$$

with respect to a probability measure $P$ defined on the Borel measurable subsets of an admissible parameter set $Q$. Here, for example, the expected state dynamics might be given by a differential equation

$$
\dot{\bar{x}}(t)=f(t, \bar{x}(t), P)
$$

where the right side is dependent on the measure $P$. In general, the function $f$ can also represent a delay or partial differential equation. Whatever the form of the system, the individual dynamics (for each $q \in Q$ ) for $x(t, q)$ are not available in the cases of interest to us here.

We specifically focus on a framework for systems of measure dependent delay differential equations that are motivated by examples arising in cellular-level modeling of HIV pathogenesis. In this case, the probability distributions characterize delays inherent in cellular pathways for virus production. These delays represent lags between acute infection of cells and the initiation of viral production as well as between productive acute infection and chronic infection (e.g., see the discussions in $[19,22,25,29,30,31,32])$. The particular model that motivates our mathematical efforts here was derived in [6] to describe infection dynamics in an in vitro experiment and is given by the system of integro-differential equations

$$
\begin{aligned}
& \dot{V}(t)=-c V(t)+n_{A} \int_{-r}^{0} A(t+\tau) d P_{1}(\tau)+n_{C} C(t)-p V(t) T(t) \\
& \dot{A}(t)=\left(r_{v}-\delta_{A}-\delta X(t)\right) A(t)-\gamma \int_{-r}^{0} A(t+\tau) d P_{2}(\tau)+p V(t) T(t) \\
& \dot{C}(t)=\left(r_{v}-\delta_{C}-\delta X(t)\right) C(t)+\gamma \int_{-r}^{0} A(t+\tau) d P_{2}(\tau) \\
& \dot{T}(t)=\left(r_{u}-\delta_{u}-\delta X(t)-p V(t)\right) T(t)+S .
\end{aligned}
$$


The compartment variables consist of the virus $V$ and the acutely infected, chronically infected, and uninfected T-cells, $A, C$, and $T$, respectively, while $X$ represents the total cell population $(A+C+T)$. The probability measures $P_{1}$ and $P_{2}$ correspond, respectively, to the distributions of the two pathogenic delays discussed above. In the derivation of this model, we ascribed no subclass variability to $A$ and $T$ and consequently only the variables $C$ and $V$ are actually expected values (with respect to $P_{1}$ and $P_{2}$, respectively). All constitutive parameters $\left(c, n_{A}, n_{C}, p, r_{v}, \delta_{A}, \delta, \gamma, \delta_{C}\right.$, $\left.r_{u}, \delta_{u}\right)$ and details of the model as well as its derivation are fully described in [6].

The example motivating our efforts here is merely one of several important examples of measure dependent dynamics that have arisen in recent applications. Realistic models in viscoelasticity of polymers and rubber as well as polarization in dielectric materials share certain features with our formulation. Specifically, recent studies $[12,13]$ of molecular-based stick-slip reptation models for heterogeneous viscoelastic polymer chain materials involve systems of the form

$$
\gamma \frac{\partial^{2} u(t, x)}{\partial t^{2}}-\frac{\partial}{\partial x} \sigma(t, x ; P)=F(t),
$$

where $u$ is the tensile displacement, $\sigma$ is the measure dependent stress

$$
\sigma(t, x ; P)=g_{e}(\varepsilon(t), \dot{\varepsilon}(t))+v \int_{\mathcal{T}} \varepsilon_{1}(t, x ; \tau) d P(\tau)
$$

with strain $\varepsilon=\frac{\partial u}{\partial x}$ and "internal”" strain $\varepsilon_{1}$ defined by

$$
\frac{\partial \varepsilon_{1}}{\partial t}(t, x ; \tau)+\frac{1}{\tau} \varepsilon_{1}(t, x ; \tau)=\dot{\varepsilon}(t, x) h(\varepsilon(t, x))
$$

In [14] it is shown that similar models (with different nonlinearities in (1.6), (1.7)) are important if one replaces Fung kernels [21] with equations for distributed molecular mechanisms in describing shear response in biotissue. In another important application [8], the systems are the usual Maxwell's equations for the electromagnetic fields $E$ and $H$ in a heterogeneous dielectric and are given by

$$
\begin{aligned}
\nabla \times E & =-\frac{\partial B}{\partial t} & \nabla \cdot D=0 \\
\nabla \times H & =\frac{\partial D}{\partial t}+J & \nabla \cdot H=0 \\
D & =\varepsilon_{r} E+\mathcal{P}, &
\end{aligned}
$$


with the exception that the probability measure $(P)$ dependent macroscopic polarization $\mathcal{P}$ is given by

$$
\mathcal{P}(t)=\int_{\mathcal{T} \times \mathcal{D}}\left[p_{1}(t ; \tau)+p_{2}(t ; d)\right] d P(\tau, d) .
$$

In this case, the microscopic orientational (Debye) polarization $p_{1}(t ; \tau)$ is defined by

$$
\dot{p}_{1}+\frac{1}{\tau} p_{1}=\tilde{\varepsilon} E
$$

and the microscopic electronic (Lorentz) polarization $p_{2}(t ; d)$ is defined by

$$
m \ddot{p}_{2}+c \dot{p}_{2}+k p_{2}=\hat{\varepsilon} E
$$

with $d=\frac{2 m}{c}$. In the equations for both $p_{1}$ and $p_{2}$, the parameters $\tau$ and $d$ represent relaxation parameters which may vary over the admissible set $\mathcal{T} \times \mathcal{D}$ according to some unknown but sought after probability measure $P=P(\tau, d)$.

In each of these examples, one seeks to characterize the material behavior to perturbations by finding a measure $P^{*}$ that provides the "best" mathematical system response when compared to observations of the physical system.

Finally, a more classical use of measure dependent dynamical systems can be found in the literature on "relaxed" or "sliding regime" control systems (e.g., [28, 35, 36] as well as in Preisach models for hysteresis in materials (e.g., see [10, 11, 24, 27, 34] and the references therein).

In this paper, we formulate an inverse problem framework for estimating the measures (using system observations) in systems of probability measure-dependent nonlinear delay differential equations. To do this, we employ the Prohorov metric (equivalent to weak convergence of measures) in a functional analytic setting. We also show how to develop an approximation approach that is readily implemented to obtain computational methods. Finally, we illustrate that the HIV systems in [6] are concrete realizations of systems satisfying the hypotheses in our framework. We thus provide a theoretical foundation for the computational methods used with experimental data in [6] as well as provide a general framework for a class of systems arising in other applications. 


\section{Theoretical Framework for General Delay System}

2.1. Theoretical Framework. We begin by considering an $n$-dimensional generalized delay system:

$$
\begin{aligned}
\dot{x}(t) & =g\left(x(t), x_{t}, P_{1}, P_{2}, \ldots, P_{n_{p}}\right), t \geq 0 \\
\left(x(0), x_{0}\right) & =(\Phi(0), \Phi),
\end{aligned}
$$

for the piecewise absolutely continuous (PAC) initial condition $\Phi$, defined on the hysteretic domain $Q=[-r, 0]$ where $r>0$. Following the standard practice in the delay differential equation literature, we let the notation $x_{t}$ represent the function $\theta \mapsto x(t+\theta),-r \leq \theta \leq 0$. Furthermore, we let $\mathbb{S}$ be the class of all Borel subsets of $Q$ (the Borel $\sigma$-algebra), and $\mathscr{P}(Q)$ be the space of probability measures on $(Q, \mathbb{S})$. In order to make this framework applicable to realistic problems, we restrict the space of admissible probability distributions $\mathscr{P}_{a d}(Q)$ to PAC functions with a finite number of saltations, where $P_{i} \in \mathscr{P}_{a d}(Q), i=1,2, \ldots, n_{p}$. To establish the theoretical framework necessary to estimate a finite number $n_{p}$ of probability distributions $P_{i}, i=1, \ldots, n_{p}$, in $(2.1)$, we define the set

$$
\Pi_{a d}=\prod_{i=1}^{n_{p}} \mathscr{P}_{a d}(Q) .
$$

Thus $\pi \in \Pi_{a d}$ means that $\pi=\left(P_{1}, \ldots, P_{n_{p}}\right)$, for $P_{1}, \ldots, P_{n_{p}} \in \mathscr{P}_{a d}(Q)$.

In the following discussion, we make use of a construct from advanced probability theory, the Prohorov metric (denoted by $\rho$ ). As we note below, convergence in the Prohorov metric is equivalent to "weak convergence of measures" (which is actually weak* convergence when considering $\mathscr{P} \subset \mathscr{C}^{*}$, where $\mathscr{C}$ is the space of continuous functions on $Q$ with the max norm). We recall the definition of the Prohorov metric on $\mathscr{P}(Q)$ for any arbitrary complete metric space $Q$ with metric dist. For any closed $F \subset Q$ and $\varepsilon>0$, we define the $\varepsilon$-neighborhood of $F$ by

$$
F^{\varepsilon}=\{q \in Q: \operatorname{dist}(\tilde{q}, q)<\varepsilon, \text { for some } \tilde{q} \in F\}
$$


The Prohorov metric is then the mapping $\rho: \mathscr{P}(Q) \times \mathscr{P}(Q) \rightarrow \mathbb{R}^{+}$defined by

$$
\rho\left(P_{1}, P_{2}\right)=\inf \left\{\varepsilon>0: P_{1}(F) \leq P_{2}\left(F^{\varepsilon}\right)+\varepsilon, \text { F closed, } F \subset Q\right\}
$$

It is known (see $[16,17,20,26,33])$ that convergence $P_{n} \rightarrow P$ in the Prohorov metric is equivalent to the statement

$$
\int_{Q} \phi d P_{n} \rightarrow \int_{Q} \phi d P \text { for all } \phi \in \mathscr{C}(Q)
$$

which is convergence in expectation or distribution, also called weak convergence of measures. We define a topology for $\Pi_{a d}$ by extending the Prohorov metric such that for $\pi, \tilde{\pi} \in \Pi_{a d}$, we let

$$
\rho_{\Pi}(\pi, \tilde{\pi})=\sum_{i=1}^{n_{p}} \rho\left(P_{i}, \tilde{P}_{i}\right)
$$

where $\pi=\left(P_{1}, \ldots, P_{n_{p}}\right)$ and $\tilde{\pi}=\left(\tilde{P}_{1}, \ldots, \tilde{P}_{n_{p}}\right)$. Given our data $d_{i} \in \mathbb{R}^{n_{d}}, n_{d} \leq n$, sampled at discrete times $t_{i}, i=1,2, \ldots, n_{t}$ and observation matrix $C \in \mathbb{R}^{n_{d} \times n}$, the goal of the inverse problem is to find a solution to

$$
\min _{\pi \in \Pi_{a d}} J(\pi ; \mathbf{d})=\min _{\pi \in \Pi_{a d}} \sum_{i=1}^{n_{t}}\left|C x\left(t_{i}, \pi\right)-d_{i}\right|^{2}
$$

where $x$ is the solution to (2.1)-(2.2) corresponding to $\pi$ and $\mathbf{d}=\left\{d_{1}, d_{2}, \ldots d_{n_{t}}\right\}$. Note that in general, $J$ need not have a unique minimizer, and thus the corresponding solution (denoted by $\left.\Pi^{*}(\mathbf{d})\right)$ could be a set of probability distribution functions. In this case, we would then define the distance between two of these sets $\Pi^{*}(\mathbf{d})$ and $\Pi^{*}(\tilde{\mathbf{d}})$ (for data $\left.\mathbf{d}, \tilde{\mathbf{d}}\right)$ to be

$$
d_{H}\left(\Pi^{*}(\mathbf{d}), \Pi^{*}(\tilde{\mathbf{d}})\right)=\inf \left\{\rho_{\Pi}(\pi, \tilde{\pi}): \pi \in \Pi^{*}(\mathbf{d}), \tilde{\pi} \in \Pi^{*}(\tilde{\mathbf{d}})\right\}
$$

which is the well-known Hausdorff distance (see [23]).

To establish the well-posedness of (2.3) within our framework, we now examine the forward and inverse problems. 
2.2. Forward Problem. To prove the well-posedness of the forward problem, we need to establish conditions for the existence and uniqueness of a solution to (2.1)-(2.2) as well as its continuous dependence upon $\pi$ in the $\rho_{\Pi}$ metric. We first give conditions on the function $g$ in (2.1).

Condition 2.1. Let $(\eta, \phi, \pi),(\tilde{\eta}, \tilde{\phi}, \tilde{\pi}) \in \mathbb{R}^{n} \times \operatorname{PAC}\left(Q ; \mathbb{R}^{n}\right) \times \Pi_{a d}$. We require that the function $g: \mathbb{R}^{n} \times \operatorname{PAC}\left(Q ; \mathbb{R}^{n}\right) \times \Pi_{a d} \rightarrow \mathbb{R}^{n}$, satisfy

$$
|g(\eta, \phi, \pi)-g(\tilde{\eta}, \tilde{\phi}, \tilde{\pi})| \leq K_{L}\left\{|\eta-\tilde{\eta}|+|\phi-\tilde{\phi}|_{\infty}\right\}+\mathscr{T}(\phi ; \pi, \tilde{\pi})
$$

where $K_{L}>0,|\cdot|,|\cdot|_{\infty}$ are the Euclidean norm in $\mathbb{R}^{n}$ and the max norm on $\operatorname{PAC}\left(Q ; \mathbb{R}^{n}\right)$, respectively, and the last term on the right side $\mathscr{T}(\phi ; \pi, \tilde{\pi})$ is some function such that for each $\phi \in \operatorname{PAC}\left(Q ; \mathbb{R}^{n}\right)$, $\mathscr{T}(\phi ; \pi, \tilde{\pi}) \rightarrow 0$ as $\rho_{\Pi}(\pi, \tilde{\pi}) \rightarrow 0$ and $|\mathscr{T}(\phi ; \pi, \tilde{\pi})| \leq K|\phi|_{\infty}$.

Following the standard Picard iteration arguments for proving the existence and uniqueness of the solution to an ordinary differential equation on a finite interval $I=\left[0, t_{f}\right]$, we begin by rewriting (2.1)-(2.2) as

$$
x(t)=\left\{\begin{array}{cc}
\Phi(0)+\int_{0}^{t} g\left(x(s), x_{s}, \pi\right) d s & \text { for } \quad t \in I \\
\Phi(t) & \text { for } t \in Q,
\end{array}\right.
$$

with initial condition $\Phi$. In the subsequent proof, we will make use of the following definition.

Definition 2.2. Let successive approximations to the solution of $(2.4)$ on $\left[-r, t_{f}\right]$ be defined for $j=0,1,2, \ldots$, as

$$
\begin{aligned}
y^{0}(t) & = \begin{cases}\Phi(0) & \text { for } t \in I \\
\Phi(t) & \text { for } t \in Q\end{cases} \\
y^{j+1}(t) & = \begin{cases}\Phi(0)+\int_{0}^{t} g\left(y^{j}(s), y_{s}^{j}, \pi\right) d s & \text { for } t \in I \\
\Phi(t) & \text { for } t \in Q\end{cases}
\end{aligned}
$$

with initial condition $\Phi$ and $\pi \in \Pi_{a d}$. 
Theorem 2.3. Consider the system of equations (2.1) with initial condition $\Phi \in P A C\left(Q ; \mathbb{R}^{n}\right)$ and $t$ in a finite interval $I=\left[0, t_{f}\right], 0<t_{f}<\infty$. If the function $g$ satisfies Condition 2.1, then there exists a unique solution to (2.1) on I.

Proof. The general idea of our argument, which is quite standard, is to show that the successive approximations defined in (2.5) converge to a unique solution of (2.1).

Let the residual function of two functions $x_{t}, \tilde{x}_{t}$ be defined as

$$
e\left(t ; x_{t}, \tilde{x}_{t}\right)=|x(t)-\tilde{x}(t)|+\left|x_{t}-\tilde{x}_{t}\right|_{\infty}
$$

for $x_{t}, \tilde{x}_{t} \in \operatorname{PAC}\left(Q ; \mathbb{R}^{n}\right), t \in I$.

If we consider the residual for the functions $y^{j+1}$ and $y^{j}$, as defined in (2.5) we find that for $t \in I$ and $j>0$

$$
\begin{aligned}
e\left(t ; y_{t}^{j+1}, y_{t}^{j}\right)= & \left|\int_{0}^{t}\left\{g\left(y^{j}(s), y_{s}^{j}, \pi\right)-g\left(y^{j-1}(s), y_{s}^{j-1}, \pi\right)\right\} d s\right| \\
& +\left|\int_{0}^{t+\cdot}\left\{g\left(y^{j}(s), y_{s}^{j}, \pi\right)-g\left(y^{j-1}(s), y_{s}^{j-1}, \pi\right)\right\} d s\right|_{\infty} \\
\leq & K_{L} \int_{0}^{t} e\left(s ; y_{s}^{j}, y_{s}^{j-1}\right) d s+\left|K_{L} \int_{0}^{t+\cdot} e\left(s ; y_{s}^{j}, y_{s}^{j-1}\right) d s\right|_{\infty}
\end{aligned}
$$

and thus

$$
e\left(t ; y_{t}^{j+1}, y_{t}^{j}\right) \leq 2 K_{L} \int_{0}^{t} e\left(s ; y_{s}^{j}, y_{s}^{j-1}\right) d s
$$

Note that the case for $j=0$ (with $t \in I$ ) is special:

$$
\begin{aligned}
e\left(t ; y_{t}^{1}, y_{t}^{0}\right) & =\left|y^{1}(t)-y^{0}(t)\right|+\left|y_{t}^{1}-y_{t}^{0}\right|_{\infty} \\
& =\left|\int_{0}^{t} g\left(y^{0}(s), y_{s}^{0}, \pi\right) d s\right|+\left|\int_{0}^{t+\cdot} g\left(y^{0}(s), y_{s}^{0}, \pi\right) d s\right|_{\infty} \\
& \leq 2 \int_{0}^{t}\left\{K_{L}\left|e\left(s, y_{s}^{0}, 0\right)\right|\right\} d s \\
& \leq 2 K_{L} \int_{0}^{t}\left(|\Phi(s)|+|\Phi|_{\infty}\right) d s \\
& \leq 2 K_{L}\left(|\Phi(0)|+|\Phi|_{\infty}\right) t
\end{aligned}
$$


and thus

$$
e\left(t ; y_{t}^{1}, y_{t}^{0}\right) \leq K_{G} t
$$

where

$$
K_{G}=2 K_{L}\left(|\Phi(0)|+|\Phi|_{\infty}\right)
$$

We claim that from (2.7) and the $j=0$ case, we have

$$
e\left(t ; y_{t}^{j+1}, y_{t}^{j}\right) \leq \frac{K_{G}}{2 K_{L}} \frac{\left(2 K_{L} t\right)^{j+1}}{(j+1) !}, t \in I
$$

Clearly, this is true for $j=0$, and the general case follows easily from induction using (2.7). Using the estimate (2.8), we can then infer that

$$
\begin{aligned}
\sum_{j=0}^{\infty} e\left(t ; y_{t}^{j+1}, y_{t}^{j}\right) & \leq \frac{K_{G}}{2 K_{L}} \sum_{j=0}^{\infty} \frac{\left(2 K_{L} t\right)^{(j+1)}}{(j+1) !} \\
& \leq \frac{K_{G}}{2 K_{L}} \mathrm{e}^{2 K_{L} t} .
\end{aligned}
$$

Thus, by the comparison test, $\sum_{j=0}^{\infty} e\left(t ; y_{t}^{j+1}, y_{t}^{j}\right)$ converges uniformly for $t \in I$, which proves that $\left\{y^{j}(t)\right\}$ converges uniformly for all $t \in I$. Denote $\lim _{j \rightarrow \infty} y^{j}(t)$ as $y(t)$. Since the $y^{j}$ 's are continuous and converge uniformly to $y$, we see that $y$ is both continuous on $I$ and satisfies (2.1) by taking limits in (2.5). Note that this also yields $y$ absolutely continuous on $I$.

To prove the uniqueness of our solution, suppose we have two distinct solutions $\{y, \tilde{y}\} \in \mathscr{C}(I)$ to (2.1). Using the same arguments as in establishing (2.7), we have

$$
\begin{aligned}
e\left(t ; y_{t}, \tilde{y}_{t}\right) \leq & \left.\mid \int_{0}^{t}\left\{g\left(y(s), y_{s}, \pi\right)-g\left(\tilde{y}(s), \tilde{y}_{s}, \pi\right)\right)\right\} d s \mid \\
& \left.+\mid \int_{0}^{t+\cdot}\left\{g\left(y(s), y_{s}, \pi\right)-g\left(\tilde{y}(s), \tilde{y}_{s}, \pi\right)\right)\right\}\left.d s\right|_{\infty} \\
\leq & 2 K_{L} \int_{0}^{t} e\left(s ; y_{s}, \tilde{y}_{s}\right) d s .
\end{aligned}
$$

Thus by Gronwall's inequality we have that

$$
|y(t)-\tilde{y}(t)|+\left|y_{t}-\tilde{y}_{t}\right|_{\infty} \leq 0 \quad \text { for } t \in I .
$$


Thus $y(t)=\tilde{y}(t)$ for $t \in I$ and also for $t \in Q$ since both solutions satisfy the same initial condition.

We have therefore now proven that there exists a unique solution (for $t \in\left[-r, t_{f}\right]$ ) to (2.4) and thus to (2.1), and that the solution is in fact absolutely continuous on $I$.

Remark 2.4. Existence and uniqueness can be established on any finite interval $I$ under somewhat weaker conditions than the global Lipschitz requirement in Condition 2.1. In particular, under conditions of local Lipschitz plus affine growth domination at infinity, one can also establish the existence and uniqueness results of Theorem 2.3. For examples and details of such arguments in the case of general nonlinear systems, see $[1,2,15]$.

In order to consider calculating a solution to the inverse problem (2.3), we must establish the well-posedness of this optimization problem, which involves both proving that the solution to (2.1) is continuous in $\pi$ and that there exists a solution to (2.3). Following the development in [7], with inspiration from $[4,5]$, we will do so by examining both problem stability and method stability.

We say that the forward problem is well-posed if the unique solution to the model (in our case $x$ ) is continuously dependent upon the measures $\pi$. Let us fix $t \in I$ and consider the continuity of a solution to (2.1)-(2.2) with respect to $\pi$. Thus we interpret the solution $x$ as the mapping $x(t, \cdot): \Pi_{a d} \rightarrow \mathbb{R}^{n}$, parameterized by the time $t$.

Lemma 2.5. If $t \in I=\left[0, t_{f}\right], Q=[-r, 0], \pi \in \Pi_{a d}$, and the right side $g$ of (2.1) satisfies Condition 2.1, then the unique solution to (2.1)-(2.2) is point-wise continuous at $\pi \in \Pi_{a d}$.

Proof. Since $\rho$ is a metric topology, it suffices to argue that $x\left(t, \pi_{i}\right) \rightarrow x(t, \pi)$ for any sequence $\left\{\pi_{i}\right\}_{i=1}^{\infty}$ where as $i \rightarrow \infty, \pi_{i} \rightarrow \pi$ in $\rho_{\Pi}$. From the definition of solutions, we find

$$
\left|x\left(t, \pi_{i}\right)-x(t, \pi)\right| \leq \int_{0}^{t}\left|g\left(x\left(s, \pi_{i}\right), x_{s}\left(\pi_{i}\right), \pi_{i}\right)-g\left(x(s, \pi), x_{s}(\pi), \pi\right)\right| d s
$$

Since $g$ satisfies Condition 2.1, we have that

$$
\left|x\left(t, \pi_{i}\right)-x(t, \pi)\right| \leq \int_{0}^{t} K_{L} e\left(s ; x_{s}\left(\pi_{i}\right), x_{s}(\pi)\right) d s+\widetilde{\mathscr{T}}\left(\pi_{i}, \pi\right),
$$


where $e$ is the residual as defined in $(2.6)$ and $\widetilde{\mathscr{T}}\left(\pi_{i}, \pi\right)=\int_{0}^{t_{f}} \mathscr{T}\left(x_{s}(\pi) ; \pi_{i}, \pi\right) d s$. Next, consider

$$
\begin{aligned}
\left|x_{t}\left(\pi_{i}\right)-x_{t}(\pi)\right|_{\infty} & \leq\left|\int_{0}^{t+\cdot}\right| g\left(x\left(s, \pi_{i}\right), x_{s}\left(\pi_{i}\right), \pi_{i}\right)-g\left(x(s, \pi), x_{s}(\pi), \pi\right)|d s|_{\infty} \\
& \leq \int_{0}^{t}\left|g\left(x\left(s, \pi_{i}\right), x_{s}\left(\pi_{i}\right), \pi_{i}\right)-g\left(x(s, \pi), x_{s}(\pi), \pi\right)\right| d s,
\end{aligned}
$$

which has the same bound as in (2.9). By combining these bounds we find that

$$
\begin{aligned}
e\left(t ; x_{t}\left(\pi_{i}\right), x_{t}(\pi)\right) \leq & 2 K_{L} \int_{0}^{t} e\left(s ; x_{s}\left(\pi_{i}\right), x_{s}(\pi)\right) d s \\
& +2 \widetilde{\mathscr{T}}\left(\pi_{i}, \pi\right),
\end{aligned}
$$

and an application of Gronwall's inequality yields

$$
\begin{aligned}
e\left(t ; x_{t}\left(\pi_{i}\right), x_{t}(\pi)\right) & \leq 2 \widetilde{\mathscr{T}}\left(\pi_{i}, \pi\right) e^{\int_{0}^{t} 2 K_{L} d s} \\
& \leq 2 \widetilde{\mathscr{T}}\left(\pi_{i}, \pi\right) e^{2 K_{L} t_{f}}
\end{aligned}
$$

From this it immediately follows that $\left|x\left(t, \pi_{i}\right)-x(t, \pi)\right| \rightarrow 0$ as $i \rightarrow \infty$ and $\pi_{i} \rightarrow \pi$ in the Prohorov metric. Therefore, we have pointwise continuity of the solution $x$ (and thus $J$ ) with respect to the optimization variable $\pi$ of interest in (2.3).

2.3. Inverse Problem Stability and Method Stability. As mentioned at the beginning of the last section, in order to fully justify our claim regarding the well posedness of the inverse problem, we need to examine questions concerning the existence of a solution to (2.3) as well as the dependence of those solutions upon given data.

Theorem 2.6. There exists a solution to the inverse problem as described in (2.3).

Proof. From results in [17], we know that if $Q$ is compact, $\left(\mathscr{P}_{a d}(Q), \rho\right)$ is compact and thus by extension, $\left(\Pi_{a d}, \rho_{\Pi}\right)$ is also compact. It is well known that a continuous function on a compact set in a metric space attains a maximum and a minimum. By Lemma 2.5 , we have that $\pi \mapsto x(t, \pi)$, for fixed $t \in I$, is continuous and thus $J$ is continuous with respect to $\pi$. We can, therefore, conclude that there exist minimizers for the cost functional $J$. 
Since the original inverse problem involves minimizing over infinite dimensional sets $\mathscr{P}(Q)$, pursuing this optimization is clearly infeasible without some type of finite dimensional approximation. We thus need to describe some approximation spaces over which the optimization becomes computationally tractable. Let $Q_{M}=\left\{q_{j}^{M}\right\}_{j=1}^{M}$ be partitions of $Q=[-r, 0]$ for $M=1,2, \ldots$ and

$$
Q_{D}=\bigcup_{M=1}^{\infty} Q_{M}
$$

where the sequences are chosen such that $Q_{D}$ is dense in $Q$. For a positive integer $M$, let

$$
\begin{gathered}
\Pi_{a d}^{M}=\left\{\pi \in \Pi_{a d}: \pi=\left(\sum_{j=1}^{M} p_{i j} \Delta_{q_{j}^{M}}, \ldots, \sum_{j=1}^{M} p_{n_{p} j} \Delta_{q_{j}^{M}}\right),\right. \\
\left.q_{j}^{M} \in Q_{M}, 0 \leq p_{i j}, \sum_{j=1}^{M} p_{i j}=1, i=1, \ldots, n_{p}\right\},
\end{gathered}
$$

where $\Delta_{q}$ is the Dirac delta-measure with atom at $q$. That is, $\Delta_{q}(E)$ is 1 if $q \in E$ and 0 if $q \notin E$. Finally, we define

$$
\Pi_{D}=\bigcup_{M=1}^{\infty} \Pi_{a d}^{M},
$$

and use the fact that $Q$ is a complete, separable metric space. By Theorem 3.1 from [5], we have that $\Pi_{D}$ is dense in $\Pi_{a d}$ in the $\rho_{\Pi}$ metric. We can, therefore, directly conclude that any element $\pi \in \Pi_{a d}$ can be approximated by a sequence $\left\{\pi_{M_{j}}\right\}, \pi_{M_{j}} \in \Pi_{a d}^{M_{j}}$ such that as $M_{j} \rightarrow \infty$, $\rho_{\Pi}\left(\pi_{M_{j}}, \pi\right) \rightarrow 0$.

Following the discussion concerning Theorem 4.1 in [5], we now state our theorem regarding the continuous dependence of the inverse problem upon the given data as well as stability under approximation of the parameter sets $\Pi_{a d}$.

Theorem 2.7. Let $Q=[-r, 0]$, assume that for fixed $t \in\left[0, t_{f}\right], \pi \mapsto x(t, \pi)$ is continuous on $\Pi_{a d}$, and let $Q_{D}$ be a countable dense subset of $Q$ as defined above. Suppose that the observed data $\mathbf{d}_{m}, \mathbf{d} \in \mathbb{R}^{n_{t} \times n_{d}}, n_{d} \leq n$, are such that $\mathbf{d}_{m} \rightarrow \mathbf{d}$ as $m \rightarrow \infty$. Moreover, suppose that $\Pi^{* M}\left(\mathbf{d}_{m}\right)$ is the set of minimizers for $J\left(\pi ; \mathbf{d}_{m}\right)$ over $\pi \in \Pi_{a d}^{M}$ corresponding to the data $\mathbf{d}_{m}$. Similarly, suppose that $\Pi^{*}(\mathbf{d})$ is the set of minimizers of $J(\pi ; \mathbf{d})$ over $\pi \in \Pi_{a d}$ corresponding to the data $\mathbf{d}$. Then, $d_{H}\left(\Pi^{* M}\left(\mathbf{d}_{m}\right), \Pi^{*}(\mathbf{d})\right) \rightarrow 0$ as $M, m \rightarrow \infty$. 
Proof. Using continuous dependence of solutions on $\pi$, compactness of $\Pi_{a d}$, and density of $\Pi_{D}$ in $\Pi_{a d}$, the arguments follow precisely those for Theorem 4.1 in [5]. We thus can claim that

$$
d_{H}\left(\Pi^{* M}\left(\mathbf{d}_{m}\right), \Pi^{*}(\mathbf{d})\right)=\inf \left\{\rho_{\Pi}\left(\pi_{m}^{M}, \pi\right): \pi_{m}^{M} \in \Pi^{* M}\left(\mathbf{d}_{m}\right), \pi \in \Pi^{*}(\mathbf{d})\right\}
$$

converges to zero as $M, m \rightarrow \infty$.

Combining the results of these two theorems, we can establish both that there exists a solution to the inverse problem and that it is continuously dependent (in the Hausdorff sense) upon the given data. We established well-posedness of the forward problem in $\$ 2.2$ and in this section, we have shown problem stability and a limited type of method stability (stability under approximations of the parameter sets) of our inverse problem. We can therefore conclude that our inverse problem is well-posed.

Lastly, we note that the theoretical results of this and the next section only apply to the identification of probability distributions. The extension to deterministic parameters, i.e., ones not associated with the delay, also follows readily from considering the Ordinary Least Squares (OLS) optimization over $\mathscr{Q}_{a d} \times \Pi_{a d}$ where $\mathscr{Q}_{a d}$ is the domain of admissible values for the deterministic parameters.

\section{APPROXIMATION IMPLEMENTATION}

3.1. Approximation of the Forward Problem. The system described by (2.1)-(2.2) is a special case of a more generalized equation that facilitates a discussion regarding its approximation. The full discretization development, based upon the ideas of [9], is presented in $[6,18]$ and will only be summarized here.

If we denote a generalized solution space as $Z=\mathbb{R}^{n} \times L_{2}\left(Q ; \mathbb{R}^{n}\right)$, we can define the nonlinear operator $\mathscr{A}: \mathscr{D}(\mathscr{A}) \subset Z \rightarrow Z$ by

$$
\begin{aligned}
\mathscr{D}(\mathscr{A}) & =\left\{(\phi(0), \phi) \in Z: \phi \in H^{1}\left(Q ; \mathbb{R}^{n}\right)\right\} \\
\mathscr{A}(\phi(0), \phi) & =(g(\phi(0), \phi), \dot{\phi}) .
\end{aligned}
$$


With this definition, we can generalize (2.1)-(2.2) in the form

$$
\begin{aligned}
\dot{z}(t) & =\mathscr{A} z(t) \\
z(0) & =z_{0},
\end{aligned}
$$

where $z_{0} \in Z$. As we shall see in $\S 4$ below, the system (2.1)-(2.2) is a special case of (3.1) where $z_{0}$ is restricted to $\mathbb{R}^{n} \times \operatorname{PAC}\left(Q, \mathbb{R}^{n}\right)$.

With index of approximation $N$, let $\left\{Z^{N}, \mathbb{P}^{N}, \mathscr{A}^{N}\right\}$ be our approximation scheme for (3.1) satisfying the conditions of Theorem 3.1 in [9], where $Z^{N}$ is a spline subspace of $Z, \mathbb{P}^{N}$ is the orthogonal projection of $Z$ onto $Z^{N}$, and $\mathscr{A}^{N}$ is the approximating operator $\mathscr{A}^{N}=\mathbb{P}^{N} \mathscr{A} \mathbb{P}^{N}$. Thus, using $\left\{Z^{N}, \mathbb{P}^{N}, \mathscr{A}^{N}\right\}$ we can generate an approximation to the formulation described by (3.1), which we denote by

$$
\begin{aligned}
\dot{z}^{N}(t) & =\mathscr{A}^{N} z^{N}(t) \\
z^{N}(0) & =\mathbb{P}^{N} z_{0} .
\end{aligned}
$$

An alternative description of (3.2) is

$$
z^{N}(t)=\mathbb{P}^{N} z_{0}+\int_{0}^{t}\left\{\mathscr{A}^{N} z^{N}(s)\right\} d s
$$

Theorem 3.1. Given the systems described in (2.1)-(2.2) and (3.2) with $z_{0}=(\Phi(0), \Phi), \Phi \in$ $\operatorname{PAC}\left(Q ; \mathbb{R}^{n}\right)$, under Condition 2.1, we have that $z^{N}(t) \rightarrow\left(x(t), x_{t}\right)$, as $N \rightarrow \infty$, uniformly in $t$ on the finite interval I, where $x$ is the solution of (2.1)-(2.2).

Proof. Following the arguments for Theorem 2.2 in [3] we readily obtain our desired conclusion.

For the full details, including the selection of an appropriate basis for $Z^{N}$, we direct the interested reader to $[6,18]$ where piecewise linear splines were succes sfully employed.

3.2. Approximation of the Inverse Problem. Since we do not have direct access to an exact solution to (2.1)-(2.2), our parameter estimation efforts are actually focused on solving

$$
\min _{\pi \in \Pi_{a d}} J^{N}(\pi, \mathbf{d})=\min _{\pi \in \Pi_{a d}} \sum_{i=1}^{n_{t}}\left|C x^{N}\left(t_{i}, \pi\right)-\mathbf{d}_{i}\right|^{2}
$$


with $n_{t}$ data observations. As described in the previous section, we can obtain a uniformly convergent (in $t$ on finite intervals for fixed $\pi$ ) numerical scheme which generates an approximate solution $x^{N}$. We have not yet shown, however, that as $N \rightarrow \infty, x^{N}\left(\pi_{i}\right) \rightarrow x(\pi)$ as $\pi_{i} \rightarrow \pi$ in the $\rho_{\Pi}$ metric, which is needed for general "method stability" (see [5]). Arguments similar to those used in the proof of Lemma 2.5 can be used to show that for fixed $N$, the approximations $x^{N}$ (and hence $J^{N}$ ) are continuous in $\pi$ on $\Pi_{a d}$. However, the convergence of $\left|x^{N}\left(t, \pi_{i}\right)-x^{N}(t, \pi)\right| \rightarrow 0$ as $N, i \rightarrow \infty$ is not as obvious (but still true), and is proven in the following lemma.

Lemma 3.2. Let $t \in I, \pi \in \Pi_{a d}$, and $\left\{\pi_{i}\right\} \in \Pi_{a d}$ be such that $\lim _{i \rightarrow \infty} \rho_{\Pi}\left(\pi_{i}, \pi\right)=0$. Then if $x^{N}\left(t, \pi_{i}\right)$ is the solution to (3.2) under the conditions of Theorem 3.1, we have $\left|x^{N}\left(t, \pi_{i}\right)-x^{N}(t, \pi)\right| \rightarrow 0$ as $N, i \rightarrow \infty$, uniformly in $t$ on $I$.

Proof. Denote $\mathbb{P}_{1}^{N}$ as the first component of the orthogonal projection operator $\mathbb{P}^{N}$ of $Z$ onto $Z^{N}$. By using arguments similar to those employed in deriving inequality (2.7), we find that for $t \in\left[0, t_{f}\right]$

$$
\begin{aligned}
e\left(t, x_{t}^{N}\left(\pi_{i}\right), x_{t}^{N}(\pi)\right) & =\left|x^{N}\left(t, \pi_{i}\right)-x^{N}(t, \pi)\right|+\left|x_{t}^{N}\left(\pi_{i}\right)-x_{t}^{N}(\pi)\right|_{\infty} \\
& \leq 2\left|x_{t}^{N}\left(\pi_{i}\right)-x_{t}^{N}(\pi)\right|_{\infty} \\
& \leq 2\left|\int_{0}^{t+} \mathbb{P}_{1}^{N} g\left(x^{N}\left(s, \pi_{i}\right), x_{s}^{N}\left(\pi_{i}\right), \pi_{i}\right)-\mathbb{P}_{1}^{N} g\left(x^{N}(s, \pi), x_{s}^{N}(\pi), \pi\right) d s\right|_{\infty} \\
& \leq 2 \int_{0}^{t}\left|g\left(x^{N}\left(s, \pi_{i}\right), x_{s}^{N}\left(\pi_{i}\right), \pi_{i}\right)-g\left(x^{N}(s, \pi), x_{s}^{N}(\pi), \pi\right)\right| d s,
\end{aligned}
$$

and since $g$ satisfies Condition 2.1, we then know that

$$
e\left(t, x_{t}^{N}\left(\pi_{i}\right), x_{t}^{N}(\pi)\right) \leq 2 K_{L} \int_{0}^{t} e\left(t, x_{s}^{N}\left(\pi_{i}\right), x_{s}^{N}(\pi)\right) d s+\widetilde{\mathscr{T}}^{N}\left(\pi_{i}, \pi\right),
$$

where $\widetilde{\mathscr{T}^{N}}\left(\pi_{i}, \pi\right)=\int_{0}^{t_{f}} \mathscr{T}\left(x_{s}^{N}(\pi) ; \pi_{i}, \pi\right) d s$. By the boundedness of $\left|x_{t}^{N}(\pi)\right|_{\infty}$ and dominated convergence, we have that $\widetilde{\mathscr{T}^{N}}\left(\pi_{i}, \pi\right) \rightarrow 0$ as $i, N \rightarrow \infty$. A simple application of Gronwall's inequality gives us that

$$
e\left(t, x_{t}^{N}\left(\pi_{i}\right), x_{t}^{N}(\pi)\right) \rightarrow 0
$$

as $N, i \rightarrow \infty$ and $\pi_{i} \rightarrow \pi$ in the $\rho_{\Pi}$ metric, and thus we conclude that $\left|x^{N}\left(t, \pi_{i}\right)-x^{N}(t, \pi)\right| \rightarrow 0$ as well. Note that the convergence is actually uniform in $t$ on $I$. 
Corollary 3.3. Under the conditions specified in Lemma 3.2, we conclude that $\left|x^{N}\left(t, \pi_{N}\right)-x(t, \pi)\right| \rightarrow$ 0 as $N \rightarrow \infty$ for $\pi_{N} \rightarrow \pi$ in the $\rho_{\Pi}$ metric. As before, convergence is uniform in $t$ on $I$.

Proof. Consider

$$
\left|x^{N}\left(t, \pi_{N}\right)-x(t, \pi)\right| \leq\left|x^{N}\left(t, \pi_{N}\right)-x^{N}(t, \pi)\right|+\left|x^{N}(t, \pi)-x(t, \pi)\right|
$$

The first term converges due to Lemma 3.2, while the second term converges as a result of our numerical scheme as given in Theorem 3.1.

With this corollary, we are now prepared to examine questions concerning the existence of a solution to (2.3) and (3.3) as well as the dependence of those solutions upon given data.

Theorem 3.4. There exists a solution to the original and approximate inverse problems in (2.3) and (3.3), respectively. Moreover, one can find solutions to the family of problems in (3.3) that converge to a solution to (2.3) as $N \rightarrow \infty$.

Proof. As noted above, we have that $\left(\Pi_{a d}, \rho_{\Pi}\right)$ is compact. By Lemmas 2.9 and 3.2, we have that both $\pi \mapsto x(t, \pi)$ and $\pi \mapsto x^{N}(t, \pi)$, for fixed $t \in I$, are continuous and thus both $J$ and $J^{N}$ are continuous with respect to $\pi$. We therefore know that there exist minimizers in $\Pi_{a d}$ for the original and approximate cost functionals $J$ and $J^{N}$, respectively.

Let $\left\{\pi_{N}^{*}\right\} \in \Pi_{a d}$ be any sequence of solutions to (3.3) and $\left\{\pi_{N_{k}}^{*}\right\}$ a convergent (in $\rho_{\Pi}$ ) subsequence of minimizers (this is possible since $\Pi_{a d}$ is a compact metric space). Recall that minimizers are not necessarily unique, but one can always select a convergent subsequence of minimizers in $\Pi_{a d}$. Denote the limit (in $\rho_{\Pi}$ ) of this subsequence as $\pi^{*}$. By the minimizing properties of $\pi_{N_{k}}^{*} \in \Pi_{a d}$, we then know that

$$
J^{N_{k}}\left(\pi_{N_{k}}^{*}\right) \leq J^{N_{k}}(\pi) \text { for all } \pi \in \Pi_{a d}
$$

By Corollary 3.3, we have the convergence of $x^{N}\left(t, \pi_{N}\right) \rightarrow x(t, \pi)$ and thus $J^{N}\left(\pi_{N}\right) \rightarrow J(\pi)$ as $N \rightarrow \infty$ when $\rho_{\Pi}\left(\pi_{N}, \pi\right) \rightarrow 0$. Therefore in the limit as $N_{k} \rightarrow \infty$, the inequality in (3.4) becomes

$$
J\left(\pi^{*}\right) \leq J(\pi) \text { for all } \pi \in \Pi_{a d},
$$


with $\pi^{*}$ providing a (not necessarily unique) minimizer of (2.3).

We have proven not only that there is a solution to the original and approximate inverse problems, but also that as we increase the state space accuracy of the approximate solution, in some sense, it approaches a solution to the original inverse problem.

Following the discussion concerning Theorem 4.1 in [5], we now state our theorem regarding the continuous dependence of the inverse problem upon the given data. We note that the following theorem describes general "method stability" in problems where one approximates both the state and parameter spaces.

Theorem 3.5. Let $Q=[-r, 0]$, assume that for fixed $t \in I=\left[0, t_{f}\right], \pi \mapsto x(t, \pi)$ is continuous on $\Pi_{a d}$ in $\rho_{\Pi}, x^{N}, J^{N}$ are the approximations given in (3.2), (3.3), and let $Q_{D}$ be a countable dense subset of $Q$ as defined above. Suppose the observed data $\mathbf{d}_{m}, \mathbf{d} \in \mathbb{R}^{n_{d}}, n_{d} \leq n$ are such that $\mathbf{d}_{m} \rightarrow \mathbf{d}$ as $m \rightarrow \infty$ and that $\Pi_{N}^{* M}\left(\mathbf{d}_{m}\right)$ is the set of minimizers for $J^{N}\left(\pi ; \mathbf{d}_{m}\right)$ over $\pi \in \Pi_{a d}^{M}$ corresponding to the data $\mathbf{d}_{m}$. Similarly, suppose that $\Pi^{*}(\mathbf{d})$ is the set of minimizers of $J(\pi ; \mathbf{d})$ over $\pi \in \Pi_{a d}$ corresponding to the data $\mathbf{d}$. Then, $d_{H}\left(\Pi_{N}^{* M}\left(\mathbf{d}_{m}\right), \Pi^{*}(\mathbf{d})\right) \rightarrow 0$ as $N, M, m \rightarrow \infty$.

Proof. If we combine the arguments of Theorem 2.7, Theorem 3.4, and Corollary 3.3, as in Theorem 4.1 in [5], we readily can obtain

$$
d_{H}\left(\Pi_{N}^{* M}\left(\mathbf{d}_{m}\right), \Pi^{*}(\mathbf{d})\right)=\inf \left\{\rho_{\Pi}\left(\pi_{N, m}^{M}, \pi\right): \pi_{N, m}^{M} \in \Pi_{N}^{* M}\left(\mathbf{d}_{m}\right), \pi \in \Pi^{*}(\mathbf{d})\right\} \rightarrow 0,
$$

as $N, M, m \rightarrow \infty$.

With the results of these two theorems, we can claim both that there exists a solution to the inverse problem and that it is continuously dependent upon the given data. We established wellposedness of the forward problem in $\$ 2.2$, and in this section, we established method stability (under approximation of the state space and parameter space) of our inverse problem. We can, therefore, conclude general well-posedness of our inverse problem. 


\section{EXAMPLE ILlustration}

We now outline an application of this framework to the HIV system (1.1)-(1.4) of $\S 1$. For fixed $t \in I, \eta \in \mathbb{R}^{4}, \phi \in P A C\left(Q ; \mathbb{R}^{4}\right)$, and $\pi=\left(P_{1}, P_{2}\right) \in \Pi_{a d}$, consider the right side of system (1.1)-(1.4),

$$
A_{1} \eta+A_{2} f_{1}(\phi, \pi)+f_{2}(\eta)+f_{3}(t)
$$

where

$$
\begin{gathered}
A_{1}=\left[\begin{array}{cccc}
-c & 0 & n_{C} & 0 \\
0 & r_{v}-\delta_{A} & 0 & 0 \\
0 & 0 & r_{v}-\delta_{C} & 0 \\
0 & 0 & 0 & r_{u}-\delta_{u}
\end{array}\right], A_{2}=\left[\begin{array}{cccc}
0 & n_{A} & 0 & 0 \\
0 & -\gamma & 0 & 0 \\
0 & \gamma & 0 & 0 \\
0 & 0 & 0 & 0
\end{array}\right] \\
f_{1}(\phi, \pi)=\left[\begin{array}{c}
\mathscr{E}_{P_{1}}\left[\phi_{2}\right] \\
\mathscr{E}_{P_{2}}\left[\phi_{2}\right] \\
\mathscr{E}_{P_{2}}\left[\phi_{2}\right] \\
0
\end{array}\right], f_{2}(\eta)=\left[\begin{array}{c}
-p \eta_{1} \eta_{4} \\
-\delta\left(\sum_{i=1}^{4} \eta_{i}\right) \eta_{2}+p \eta_{1} \eta_{4} \\
-\delta\left(\sum_{i=1}^{4} \eta_{i}\right) \eta_{3} \\
-\delta\left(\sum_{i=1}^{4} \eta_{i}\right) \eta_{4}-p \eta_{1} \eta_{4}
\end{array}\right]
\end{gathered}
$$

and

$$
f_{3}(t)=[0,0,0, S]^{T}
$$

We note that the nonlinearities exemplified by terms such as $p \eta_{1} \eta_{4}$ in $f_{2}$ are both biologically unrealistic and fail to satisfy a global Lipschitz condition. Such terms in (1.1)-(1.4) can be correctly viewed as approximations to nonlinear saturation terms. We address this issue, as we did in [6], with an approach which is readily justified from a biological viewpoint by saturation dynamics principles. We define $\bar{f}_{2}$ using $f_{2}$ with standard saturation limited nonlinearities by replacing $p \eta_{1} \eta_{4}$ 
by $p_{1}\left(\eta_{1}\right) \eta_{4}$ and $\delta \eta_{i} \eta_{j}$ by $\delta_{i}\left(\eta_{i}\right) \eta_{j}$ for $i, j=2,3,4$, where the functions $p_{1}$ and $\delta_{i}$ are defined as

$$
p_{1}\left(\eta_{1}\right)=\left\{\begin{aligned}
0 & ; \eta_{1}<0 \\
p \eta_{1} & ; 0 \leq \eta_{1} \leq \bar{\eta}_{1} \\
p \bar{\eta}_{1} & ; \bar{\eta}_{1}<\eta_{1},
\end{aligned}\right.
$$

and

$$
\delta_{i}\left(\eta_{i}\right)=\left\{\begin{aligned}
0 & ; \eta_{i}<0 \\
\delta \eta_{i} & ; 0 \leq \eta_{i} \leq \bar{\eta}_{i} \\
\delta \bar{\eta}_{i} & ; \bar{\eta}_{i}<\eta_{i}
\end{aligned}\right.
$$

(for finite upper bounds $\bar{\eta}_{i} \in \mathbb{R}^{+}, i=1,2,3,4$ ). The resulting function $\bar{f}_{2}$ is now globally Lipschitz (see [3]). With $g$ redefined for fixed $t$ as

$$
g(\eta, \phi, \pi)=A \eta+f_{1}(\phi, \pi)+\bar{f}_{2}(\eta)+f_{3}(t)
$$

the well-posedness of the inverse problem for $\pi=\left(P_{1}, P_{2}\right)$ follows if we establish that $g$ satisfies Condition 2.1. We proceed to argue that Condition 2.1 holds by observing that for $\eta, \tilde{\eta} \in \mathbb{R}^{4}$, $\phi, \tilde{\phi} \in P A C\left(Q ; \mathbb{R}^{4}\right)$, and $\pi=\left(P_{1}, P_{2}\right), \tilde{\pi}=\left(\tilde{P}_{1}, \tilde{P}_{2}\right) \in \Pi_{a d}$

$$
|g(\eta, \phi, \pi)-g(\tilde{\eta}, \tilde{\phi}, \tilde{\pi})| \leq\left|A_{1}\right||\eta-\zeta|+\left|A_{2}\right|\left|f_{1}(\phi, \pi)-f_{1}(\tilde{\phi}, \tilde{\pi})\right|+\left|\bar{f}_{2}(\eta)-\bar{f}_{2}(\tilde{\eta})\right|
$$

To bound the second term on the right side of (4.4), let us examine

$$
\begin{aligned}
\left|f_{1}(\phi, \pi)-f_{1}(\tilde{\phi}, \tilde{\pi})\right| \leq & \left|\mathscr{E}_{P_{1}}\left[\phi_{2}\right]-\mathscr{E}_{\tilde{P}_{1}}\left[\tilde{\phi}_{2}\right]\right|+2\left|\mathscr{E}_{P_{2}}\left[\phi_{2}\right]-\mathscr{E}_{\tilde{P}_{2}}\left[\tilde{\phi}_{2}\right]\right| \\
\leq & \left|\mathscr{E}_{P_{1}}\left[\phi_{2}\right]-\mathscr{E}_{\tilde{P}_{1}}\left[\phi_{2}\right]\right|+\left|\mathscr{E}_{\tilde{P}_{1}}\left[\phi_{2}\right]-\mathscr{E}_{\tilde{P}_{1}}\left[\tilde{\phi}_{2}\right]\right| \\
& +2\left|\mathscr{E}_{P_{2}}\left[\phi_{2}\right]-\mathscr{E}_{\tilde{P}_{2}}\left[\phi_{2}\right]\right|+2\left|\mathscr{E}_{\tilde{P}_{2}}\left[\phi_{2}\right]-\mathscr{E}_{\tilde{P}_{2}}\left[\tilde{\phi}_{2}\right]\right| \\
\leq & 3|\phi-\tilde{\phi}|_{\infty}+\left|\mathscr{E}_{P_{1}}\left[\phi_{2}\right]-\mathscr{E}_{\tilde{P}_{1}}\left[\phi_{2}\right]\right| \\
& +2\left|\mathscr{E}_{P_{2}}\left[\phi_{2}\right]-\mathscr{E}_{\tilde{P}_{2}}\left[\phi_{2}\right]\right| .
\end{aligned}
$$


The last two terms comprise a function $\mathscr{T}$ which, for fixed $\phi_{2}$, converges to zero when $P_{1}, P_{2}$ converge to $\tilde{P}_{1}, \tilde{P}_{2}$ in the Prohorov metric, and moreover satisfies the dominating bound of Condition 2.1. Thus we have

$$
\left|f_{1}(\phi, \pi)-f_{1}(\tilde{\phi}, \tilde{\pi})\right| \leq 3|\phi-\tilde{\phi}|_{\infty}+\mathscr{T}\left(\phi_{2} ; \pi, \tilde{\pi}\right)
$$

as required in Condition 2.1.

To bound the third term on the right side of (4.4), we refer to the arguments presented in Lemma 4.2.1 in [6]. The multidimensional Mean Value Theorem implies that for $\eta, \tilde{\eta} \in \mathbb{R}^{4}$

$$
\bar{f}_{2}(\eta)-\bar{f}_{2}(\tilde{\eta})=\int_{0}^{1}\left\langle\mathbf{D} \bar{f}_{2}(\eta+\theta(\tilde{\eta}-\eta)), \eta-\tilde{\eta}\right\rangle d \theta
$$

where the $4 \times 4$ matrix valued function is given by

$$
\mathbf{D} \bar{f}_{2}=\left[\begin{array}{llll}
\partial_{1} \bar{f}_{2} & \partial_{2} \bar{f}_{2} & \partial_{3} \bar{f}_{2} & \partial_{4} \bar{f}_{2}
\end{array}\right]
$$

where $\partial_{i} \bar{f}_{2}$ is the partial derivative of $\bar{f}_{2}$ with respect to the $i$ th component of its vector argument. By the definition of $\bar{f}_{2}$ we know that the quantity $\left|\mathbf{D} \bar{f}_{2}\right|$ is be bounded by some $K_{2}$. A simple application of Cauchy-Schwarz then yields

$$
\begin{aligned}
\left|\bar{f}_{2}(\eta)-\bar{f}_{2}(\tilde{\eta})\right| & \leq \int_{0}^{1}\left|\mathbf{D} \bar{f}_{2}(\eta+\theta(\tilde{\eta}-\eta))\right||\tilde{\eta}-\eta| d \theta \\
& \leq K_{2}|\eta-\tilde{\eta}|
\end{aligned}
$$

and the combination of this bound with (4.4) yields our claim with $K_{L}=\max \left\{3, K_{2}\right\}$.

Therefore, since the function $g$ satisfies Condition 2.1, we can conclude well-posedness of the inverse problem of identifying distributions associated with time delays in the HIV viral infection dynamics described by equations (1.1)-(1.4). 


\section{Conclusions}

Our efforts here are motivated by a class of mathematical models which partition populations into subclasses according to a characteristic temporal delay. These delays can be viewed as realizations of an associated probability distribution representing the percentage of a subclass in a population. The specific microscale structure of these populations is, however, only accessible as an expectation with respect to the distribution. In $\S 1$, we presented examples of models exhibiting these features which occur in a wide variety of fields such as immunological population dynamics, viscoelasticity of polymers and rubber, and polarization in dielectric materials. In $\S 2$, we developed a mathematical framework in which we establish existence and uniqueness of the forward problem and well-posedness for the inverse problem of estimating the probability measures. These results included method stability under numerical approximations, thus leading to a computationally feasible methodology. Finally, we verified that one motivating model of HIV infection dynamics (studied previously in [6]) satisfies all the conditions of our framework, thereby providing a theoretical foundation for inverse problem computations with these models.

\section{ACKNOWLEDGMENTS}

This research was supported in part by the Air Force Office of Scientific Research under grants AFOSR-F49620-01-1-0026 and AFOSR-FA9550-04-1-0220, in part by the Joint DMS/NIGMS Initiative to Support Research in the Area of Mathematical Biology under grant 1R016M6729901, and in part by a University of Michigan Rackham Faculty Development Grant.

Collaboration was also facilitated by author visits to the Statistical and Applied Mathematical

Sciences Institute (SAMSI), Research Triangle Park, NC which is funded by the National Science Foundation under grant DMS-0112069.

\section{REFERENCES}

[1] A. Ackleh, H. T. Banks, and G. A. Pinter. Well-posedness results for models of elastomers. J. Math. Anal. Appl., 268:440-456, 2002.

[2] H. T. Banks. Approximation of nonlinear functional differential equation control systems. Journal of Optimization Theory and Applications, 29:383-408, 1979. 
[3] H. T. Banks. Identification of nonlinear delay systems using spline methods. In V. Lakshmikantham, editor, Nonlinear Phenomena in Mathematical Sciences, pages 47-55. Academic Press, New York, NY, 1982.

[4] H. T. Banks. Incorporation of uncertainty in inverse problems. Technical Report CRSC-TR02-08, Center for Research in Scientific Computation, N. C. State University, Raleigh, August 2002. In Y. C. Han et al., editors, Recent Developments in Theories and Numerics: International Conference on Inference Problems, pages 26-36. World Scientific Press, 2003.

[5] H. T. Banks and K. L. Bihari. Modeling and estimating uncertainty in parameter estimation. Inverse Problems, 17:95-111, 2001.

[6] H. T. Banks, D. M. Bortz, and S. E. Holte. Incorporation of variability into the mathematical modeling of viral delays in HIV infection dynamics. Mathematical Biosciences, 183(1):63-91, 2003.

[7] H. T. Banks, M. W. Buksas, and T. Lin. Electromagnetic Material Interrogation Using Conductive Interfaces and Acoustic Wavefronts, volume FR21 of Frontier in Applied Mathematics. SIAM, Philadelphia, PA, 2000.

[8] H. T. Banks and N. L. Gibson. Well-posedness in Maxwell systems with distributions of polarization relaxation parameters. Technical Report CRSC-TR04-01, Center for Research in Scientific Computation, N. C. State University, Raleigh, January 2004. Applied Mathematics Letters, to appear.

[9] H. T. Banks and F. Kappel. Spline approximations for functional differential equations. Journal of Differential Equations, 34:496-522, 1979.

[10] H. T. Banks, A. J. Kurida, and G. Webb. Identification of hysteretic control influence operators representing smart actuators, Part I: Formulation. Math. Prob. in Engr., 3:287-328, 1997.

[11] H. T. Banks, A. J. Kurida, and G. Webb. Identification of hysteretic control influence operators representing smart actuators, Part II: Convergent approximations. J. Intel. Mat. Sys. and Structures, 8:536-550, 1997.

[12] H. T. Banks, N. G. Medhin, and G. A. Pinter. Multiscale considerations in modeling of nonlinear elastomers. Technical Report CRSC-TR03-42, Center for Research in Scientific Computation, N. C. State University, Raleigh, October 2003. J. Comp. Meth. Sci. and Engr., to appear.

[13] H. T. Banks, N. G. Medhin, and G. A. Pinter. Nonlinear reptation in molecular based hysteresis for polymers. Technical Report CRSC-TR03-45, Center for Research in Scientific Computation, N. C. State University, Raleigh, December 2003. Quarterly Applied Mathematics, to appear.

[14] H. T. Banks and G. A. Pinter. A probabilistic multiscale approach to hysteresis in shear wave propagation in biotissue. Technical Report CRSC-TR04-03, Center for Research in Scientific Computation, N. C. State University, Raleigh, January 2004. SIAM J. Multiscale Modeling and Simluation, to appear.

[15] H. T. Banks, H. T. Tran, and S. Wynne. A well-posedness result for a shear wave propagation model. In Control of CPS, Intl. Series Num. Math., volume 143, pages 25-40. Birkhäuser, Basel, 2002.

[16] H. Bergström. Weak Convergence of Measures. Academic Press, New York, NY, 1982. 
[17] P. Billingsley. Convergence of Probability Measures. John Wiley \& Sons, New York, NY, 1968.

[18] D. M. Bortz. Modeling, Analysis, and Estimation of an in vitro HIV Infection Using Functional Differential Equations. Ph.D. dissertation, N. C. State University, Raleigh, NC, 2002.

[19] R. V. Culshaw and S. Ruan. A delay-differential equation model of HIV infection of CD4+ T-cells. Mathematical Biosciences, 165:27-39, 2000.

[20] B. Fristedt and L. Gray. A Modern Approach to Probability Theory. Birkhäuser, Boston, MA, 1997.

[21] Y. C. Fung. Biomechanics: Mechanical Properties of Living Tissues. Springer-Verlag, New York, NY, 1993.

[22] Z. Grossman, M. Polis, M. B. Feinberg, Z. Grossman, I. Levi, S. Jankelevich, R. Yarchoan, J. Boon, F. de Wolf, J. M. A. Lange, J. Goudsmit, D. S. Dimitrov, and W. E. Paul. Ongoing HIV dissemination during HAART. Nature Medicine, 5:1099-1104, 1999.

[23] J. L. Kelley. General Topology. Van Nostrand-Reinhold, Princeton, NJ, 1955.

[24] M. A. Krasnoselskii and A. V. Pokrovskii. Systems with Hysteresis. Springer-Verlag, New York, NY, 1989.

[25] A. L. Lloyd. The dependence of viral parameter estimates on the asumed viral load life cycle: limitations of studies of viral load data. Proceedings of the Royal Society of London Series B, 268:847-854, 2001.

[26] M. Loève. Probability Theory. Van Nostrand Company, Princeton, NJ, 1960.

[27] I. D. Mayergoyz. Mathematical Models of Hysteresis. Springer-Verlag, New York, NY, 1991.

[28] E. J. McShane. Relaxed controls and variational problems. SIAM J. Control, 5:438-485, 1967.

[29] J. E. Mittler, B. Sulzer, A. U. Neumann, and A. S. Perelson. Influence of delayed viral production on viral dynamics in HIV-1 infected patients. Mathematical Biosciences, 152:143-163, 1998.

[30] P. W. Nelson, J. E. Mittler, and A. S. Perelson. Effect of drug efficacy and the eclipse phase of the viral life cycle on estimates of HIV viral dynamic parameters. Journal of Acquired Immune Deficiency Syndromes, 26:405-412, 2001.

[31] P. W. Nelson, J. D. Murray, and A. S. Perelson. A model of HIV-1 pathogenesis that includes an intracellular delay. Mathematical Biosciences, 163:201-215, 2000.

[32] P. W. Nelson and A. S. Perelson. Mathematical analysis of delay differential equation models of HIV-1 infection. Mathematical Biosciences, 179:73-94, 2002.

[33] D. W. Stroock. Probability Theory, An Analytical View. Cambridge University Press, New York, NY, 1993.

[34] A. Visintin. Differential Models of Hystersis. Springer-Verlag, New York, NY, 1994.

[35] J. Warga. Functions of relaxed controls. SIAM J. Control, 5:628-641, 1967.

[36] J. Warga. Optimal Control of Differential and Functional Equations. Academic Press, New York, NY, 1972. 